HACKETT, C.; ROSE, D.A. A model of the extension and branching of a seminal root of barley, and its use in studying relations between root dimensions. II. Results and inferences from manipulation of the model. Australian Journal of Blology Science, v.25, p.681-690, 1972b.

KLEPPER, B.; RICKMAN, R.W. Modeling crop root growth and function. Advances in Agronomy, p.113-132, 1990.

KLEPPER, B.; RICKMAN, R.W. Predicting root development of crop plants. IN: Predicting crop phenology. TOM HODGES (editor). CRC Press. 1991.

LUNGLEY, D.R. The growth of root systems - A numerical computer simulation model. Plant and Soll, v.38, p.145-159. 1973 .

MASLE, J.; PASSIOURA, J.B. The effect of soil strength on the growth of young wheat plants. Australlan Journal of Plant Physiology, v.14, p.643-656. 1987.

MASLE, J.; FARQUHAR, G.D. GIFFORD, R.M. Growth and carbon economy of wheat seedlings as affected by soil resistance to penetration and ambient partial pressure of $\mathrm{CO}_{2}$. Australian Journal of Plant Physiology, v.17, p.465-487, 1990.

NYE, P.H.; TINKER, P.B. Solute movement in the soilroot system. Studies in Ecology - Volume 4. University of California Press. 1977

PAGES, L.; JORDAN, M.O.; PICARD, D. A simulation model of the three-dimensional architecture of the maize root system. Plant and Soll, v.119, p.147-154, 1989.

PORTER, J.R.; KLEPPER, B.; BELFORD, R.K. A model (WHTROOT) which synchronizes root growth and developement with shoot development for winter wheat. Plant and Soll, v.92, p.133-145, 1986.

SIMUNEK, J.; VOGEL, T.; VAN GENUCHTEN, M.Th. The SWMS-2D code for simulating water flow and solute transport in two-dimensional variably saturated media. Research report No 126. US Salinity Laboratory, Riverside, California. 1992.

\section{EMERGING SOIL MEASUREMENT TECHNIQUES AT THE MICROSCALE}

The current understanding of water flow and contaminant transport in soils has been limited by existing measurement technologies. For example, the majority of established soil water measurement methods require an instrument to be inserted at or near the region of interest, thereby disturbing the environment being measured. Secondly, their operation allows for a limited number of 'point' measurements with each measurement representative for a soil volume which may be too small or too large, depending on the research objective. Therefore, measurements or results need to be scaled up or down so that the data can be interpreted properly for the scale of interest. In the application of the conceptual description of flow and transport we are limited to the macroscopic approach, because parameters can be measured only at the $\mathrm{cm}$-scale or larger. Macropore and bypass flow are suggested as mechanisms by which accelerated breakthrough of contaminants occurs and their study requires measurements at the microscale. To better understand mechanical dispersion, diffusion, chemical and physical adsorption, degradation, and the role of immobile soil water regions, and other dynamic systems requires poresize scale measurements.

Noninvasive, nondestructive measurement techniques are used at spatial scales of millimeters to micrometers. These methods allow observations of changing fluid phase content and solute concentration, which with increasing sophistication resolve increasingly smaller features of the pore space (Anderson \& Hopmans, 1994). We present a current review of the concepts and operation of electromagnetic (EM) radiation techniques. EM methods are categorized by those employing radiography and those based on the principle of tomography. Although both methods make use of the attenuation of electromagnetic energy by the porous medium, radiography yields integrative information across the thickness of the medium (two-dimensional), whereas tomography makes use of reconstruction algorithms which resolve the internal distribution of phase content or density (three-dimensional). Both methods are used across a spectrum of frequencies (light, $x$ - and $y$-rays).

In a radiographic measurement, electromagnetic radiation is passed through the investigated medium, with the transmitted portion quantified using a detection device. For a parallel, monoenergetic beam of $x$ or $y$-rays, the distribution of transmitted radiation through the medium is a function of the adsorption characteristics, and is defined by Lambert-Beer's law:

$$
I_{\lambda}=I_{0, \lambda} \exp \left[-\int_{0}^{x} \mu_{\lambda}^{\prime}(x) d x\right]
$$


where $I_{\lambda}$ is the transmitted radiation intensity, $I_{0, \lambda}$ is the incident electromagnetic radiation intensity, $\mu_{\lambda}{ }^{\prime}$ is the linear attenuation coefficient, with the subscript $\lambda$ (wavelength of radiation) indicating the frequency dependency of the attenuation parameter and the validity of Equation (7) for monochromatic radiation. The linear attenuation coefficient is a function of the absorbing material including its density, and $X$ indicates the thickness of the medium between the source and detector. The linear attenuation coefficient is the sum of various type of interactions of the EMradiation with the adsorbing material. These are photoelectric absorption, Compton scattering, pair production, and Rayleigh scattering. Photoelectric absorption is a function of the density of the medium, atomic and mass numbers, and wave length of the EM-radiation. It is most pronounced at the lower energies $(25 \mathrm{keV}$ for soft tissue and $500 \mathrm{keV}$ for lead), and increases rapidly with the third to fourth power of the atomic number of the absorber. The Compton scatter in soils is dominating at higher energies $(>100 \mathrm{keV})$ and relatively independent of atomic number. Rayleigh scattering can be important for very low energy $x$-rays but is of only minor importance in the typical energy range of interest. Pair production is only present at energy levels beyond our applications ( $>1 \mathrm{MeV}$ ). Thus, EM-absorption is dependent on bulk density, fluid content, and chemical composition, and therefore radiography offers possibilities to determine spatial and temporal distributions of fluid saturations and solute concentrations in multi-fluid soil systems.

Equation (7) includes the spatial dependence of the mass attenuation coefficient, reflecting the fact that the fluid or solid phase absorption characteristics may vary with space. In radiography, however, $I_{\lambda}$, is determined from the average attenuation along the path length (thickness) of the investigated medium, so that for monochromatic radiation equation (7) becomes:

$$
I_{\lambda}=I_{0} \exp \left[-\mu^{\prime} X\right]
$$

with $\mathrm{X}$ denoting the total travel path or thickness of the medium. For a medium consisting of solid, water and gas phase, and denoting the mass absorption coefficient, $\mu$, as the ratio of the linear attenuation coefficient and density, LambertBeer's equation can be rewritten as

$$
I_{\lambda}=I_{0} \exp \left[-\mu_{s} \rho X-\mu_{w} \theta \rho_{w} X\right]
$$

where $\mu_{s}$, and $\mu_{w}$ denote the mass attenuation coefficients of soil and water, respectively, and the attenuation of the gas phase is neglected. Thus, in using the radiographic technique, the collected attenuation data represent an integrated average across the soil sample. When using dualenergy gamma sources, each with different mass absorption coefficients, the above equation is applied to each source, after which the two equations can be solved to yield estimates of volumetric water content $(\theta)$ and soil bulk density $(\rho)$. Based on consideration of half-life, self absorption and distinct attenuation coefficients, radioactive sources that are used are ${ }^{241} \mathrm{Am}$ (60 $\mathrm{keV})$ and ${ }^{137} \mathrm{Cs}(662 \mathrm{keV})$. A combination of pulse-height analyzers and detectors are used to discriminate and count photons in the appropriate energy spectra. The early literature (e.g. Corey et al. 1971) described the experimental requirements. Later applications improved calibration, automation and error analysis of such dual-energy gamma radiation systems (e.g. Hopmans \& Dane, 1986), or applied dual-energy gamma to measure solute concentrations (Grismer et al., 1986; Oostrom et al. 1992) and fluid saturation in multi-phase systems (Dane et al., 1992). Using the dual-gamma radiation technique, fluid and porous medium properties can be determined noninva-sively and nondestructively. Measurements yield average values over the transmission length or sample thickness, thereby allowing two-dimensional visualization of fluid contents and media density. Optimal sample thickness varies with absorption characteristics and photon intensity, but is generally between 5 and $10 \mathrm{~cm}$. Spatial resolution is controlled by the collimation, and is generally between 1 and $10 \mathrm{~mm}$. Rapid measurements and simple calculations allow for almost immediate results.

Despite that most sources emit polychromatic $x$-rays, which are subject to preferential absorption of the lower energy photons, it is assumed that their attenuation is governed by Lambert Beer's law of absorption as well. The first application of $\mathrm{X}$-ray radiography is credited to Rontgen in 1895. Tidwell \& Glass (1994) applied this technique to image spatial and temporal distribution of fluid saturation. Their 
technique records the light output through the $x$ ray exposed film on a CCD camera, which is then digitized into a 512 by 512 points array, yielding a spatial resolution of approximately 8 pixels per $\mathrm{cm}$ in their experiments. Liquid water was doped with a 10 percent $\mathrm{KI}$ solution, thereby increasing the sensitivity of transmitted $\mathrm{x}$-ray intensity to degree of fluid saturation. In addition to the exponential absorption, visible light is scattered and refracted at fluid-fluid and fluid-solid interfaces. Therefore, in the visible light transmission method (Tidwell \& Glass, 1994), the intensity of the transmitted light is a function of the refractive indices of the light-absorptive phases. Imaging of the light transmission technique (Glass et al., 1989) is experimentally identical to the $x$-ray absorption technique, with the $\mathrm{x}$-ray film replaced by the $1 \mathrm{~cm}$ thick silica sand medium. However, the physical basis of their measurements are quite different. Whereas the attenuation of $x$-rays is used to infer fluid distribution, the light technique uses the property that light transmission is increased as the water saturation increases. Both the $\mathrm{x}$-ray method and the light transmission technique provided quantitative liquid saturation information with an accuracy of 5 percent saturation. The speed of the light transmission measurement makes it especially suitable for transient flow experiments. Both methods have demonstrated their potential in detecting preferential flow mechanisms in heterogeneous and fractured media in thin slab systems (Nicholl et al., 1994). The light transmission technique is limited since it requires a translucent porous medium with a thickness smaller than $1 \mathrm{~cm}$.

Classic radiography projects a threedimensional distribution of attenuation coefficients as a shadow on a two-dimensional plane. Therefore, it does not provide information on the variation in attenuation along the beam direction. A major breakthrough in the 1970's, the introduction of $\mathrm{x}$-ray transmission computed tomography (CT or CAT) in the medical sciences, allowed nondestructive cross-sectional imaging (Hounsfield, 1973). Using a collimated x-ray source and detector array, a series of onedimensional attenuation projections are obtained at a prescribed set of angles through the test object. These projections are analyzed using a reconstruction algorithm to yield a twodimensional map of attenuation coefficients. Three-dimensional representations are cons- tructed by stacking individually obtained crosssectional maps.

CT is based on the assumption that for each detector, the logarithm of the ratio of the emitted to the detected photon intensity is equal to the line integral over the respective beam path through the two-dimensional distribution of linear attenuation coefficients (Equation (7) for monochromatic radiation). By measuring a sufficient number of these line integrals through variation of incident angle, the distribution of attenuation values ( $\mu^{\prime}$ as a function of position) can be obtained. However, CT x-ray radiation is usually not monochromatic, but instead consists of a spectrum of photon energies (i.e., wavelengths). The polychromatic nature of $x$-rays causes a deviation from the exponential attenuation predicted by Lambert-Beer's law because the lower-energy portion of the emitted spectrum is being attenuated more strongly than the higher-energy portion as the beam passes through the object, an effect known as beamhardening'. A typical beam-hardening artifact is the presence of elevated attenuation values near the circumference of the measured object. The problem can be reduced by selective filtering of the lower energy photons. Scanners differ in number and size of sources and detectors, and type of source collimation (pencil beam, fan or cone beam), but have developed toward higher spatial resolution and faster scanning times.

A CT-image plots the two- or threedimensional spatial distribution of attenuation coefficients, with the average mass attenuation equal to the sum of the weighted mass attenuation coefficients of the constituent elements. The spatial resolution is controlled by the size of the smallest possible volume elements or voxels. Voxel size is determined by many factors including source and detector size, source photon energy and flux, detected photon flux, and acquisition time. Since, medical scanners are primarily designed for investigation of living tissue, these use relatively low energy levels ( 120 $\mathrm{keV}$ ) and low photon fluxes. Consequently their spatial resolution is limited $(500-1000$ micrometer). Industrial applications are not constrained by dose restrictions, and therefore can use $\mathrm{x}$-ray systems with higher energies ( $\mathrm{x}$-ray computed microtomography, CMT). Smaller source beam diameters and new developments in detection technology have increased the maximum spatial resolution to 5-15 micrometer 
Other developments, such as the introduction of cone-beam imaging and two-dimensional detectors which allow acquisition of a full threedimensional data sets with a single object rotation, have greatly decreased the scanning time of three-dimensional objects.

The CT-technique was quickly adopted by the oil industry to improve oil recovery and identification of drilling sites, and was first introduced in soils by Petrovic et al. (1982). Follow-up studies (e.g. Crestana et al., Anderson et al., 1988; Hopmans et al., 1992) have shown that linear relationships exist between attenuation values and the soil's dry bulk density and volumetric water content, whereas Steude et al. (1989) and Vinegar \& Wellington (1987) demonstrated the application of CT to the monitoring of transport and breakthrough of solutes. At the same time, it has also been shown that $\mathrm{x}$-ray CT can be successfully applied to image the geometry of macropores and plant roots. Recent work has demonstrated the potential for using CT in the characterization of root geometry (Tollner et al., 1994, Heeraman et al., 1997). A review of CT applications can be found in Anderson \& Hopmans (1994). Examples of the use of gamma ray CT in porous media are presented by Brown et al. (1993) and Phogat et al. (1991). The large advantage of gamma over $x-$ ray sources is that they are monochromatic. On the other hand, photon fluxes are much lower, thereby requiring proportionally larger acquisition times. The dual-energy gamma CT system as introduced by Phogat et al. (1991) allows the imaging of the spatial distributions of two soil characteristics, by the independent scanning using two gamma sources $\left({ }^{137} \mathrm{Cs}\right.$ and $\left.{ }^{169} \mathrm{Yb}\right)$. For example, it allows simultaneous measurement of the three-dimensional distribution of volumetric water content and soil density or chemical concentration and fluid content.

Although the use of cone beam geometry in $\mathrm{x}$-ray CT has further increased the spatial resolution, the three-dimensional images obtained from cone beam scanners are distorted. This is caused by the decreasing photon intensity as the $\mathrm{X}$-ray beam increases in size while passing through the medium. Synchrotron radiation sources, on the other hand, produce parallel $x$-ray beams and can be made nearly monochromatic. These two properties together eliminate the distortion effects as caused by the cone-shaped beam geometry, as well as beam-hardening because of the near constant energy of the synchrotron $\mathrm{x}$-rays. Their high brightness (ensures high photon flux density) has now provided a means to measure at a spatial resolution of $1 \mathrm{~mm}$ or better, whereas scanning times have been reduced to seconds rather than minutes, thereby providing opportunities to study transient flow and transport problems. $\mathrm{X}$-ray imaging using the synchrotron is also referred to as $X$-ray tomographic microscopy (XTM), because of the microscopic spatial resolution. Results from using in porous media have been presented by Spanne et al. (1994a, 1994b) using the Brookhaven National Synchrotron Light Source X26 beamline, and by Liu et al. (1993) at the Comell High Energy Synchrotron Source (CHESS) to measure rapidly changing soil water contents in preferential flow fields. Kinney et al. (1994) present a clear overview of the recent advances in XIM with examples obtained at the Stanford Synchrotron Radiation Laboratory. The advantages of using synchrotron x-ray sources will generate new opportunities, especially with the becoming available of new sources, such as the Advanced Photon Source at Argonne National Laboratory.

Various other emerging microscopic measurement techniques are becoming available. These include Nuclear Magnetic Resonance Imaging (NMRI), fiber-optic sensors, micromodels, miniature ion-specific electrodes, photoluminescence volumetric imaging (PVI), and micro-tensiometers. However these will not be treated here, but there description is presented in Hopmans et al. (1997).

It is clearly astonishing how fast new developments are becoming available only recently. For example, microscopic techniques offer a broad spectrum of opportunities in vadose zone research, especially in combination with noninvasive measurements (e.g., $x$-ray $C T$ and NMR) to study spatial and temporal distributions of fluid phases and their interfaces. Using such systems, models of preferential flow (macro versus matrix flow and film flow) or accelerated breakthrough (mobile versus immobile soil solution, and inter- and intra-aggregate solute transport) could be verified experimentally. The various microscopic techniques might allow simultaneous measurements of physical, chemical and biological processes, for example to study the 
interactions between plant roots and soil water and other soil environmental variables using a combination of ion- specific microelectrodes, CT and micro-tensiometric measurements.

\section{REFERENCES}

ANDERSON, S.H.; HOPMANS, J.W. Tomography of Soil-Water-Root Processes. Soil Science Society of America Special Publication Number 36. American Society of Agronomy lnc. Madison, Wisconsin, USA. 1994.

BROWN, G.O.; STONE, M.L. GAZIN, J.E. Accuracy of gamma ray computerized tomography in porous media. Water Resources Research, v.29, n.2, p.479-486, 1993.

COREY, J.C.; PETERSON, S.F.; WAKAT, M.A. Measurement of attenuation for Cs and Am gamma rays for soil density and water content determinations. Soll Science Society of America Jourmal, v.35, p.215-219, 1971.

CRESTANA, S.; MASCARENHAS, S.; POZZIMUCELLI, R.S. Static and dynamic three-dimensional studiers of water in soil using computed tomographic scanning. Soll Science, v.140, n.5, p.326-332, 1985.

DANE, J.H.; OOSTROM, M. MISSILDINE, B.C. An improved method for the determination of capillary pressure-saturation curves involving TCE, water and air. Journal of Contamination Hydrology, v.11, p.69-81. 1992.

GLASS, R.J.; STEENHUIS, T.S.; PARLANGE, J.Y. Mechansims for finger persistence in homogeneous unsaturated porous media: Theory and verification. Soll Science, v.148, p.60-70, 1989.

GRISMER, M.E.; MCWORTHER, D.B. KLUTE, A. Monitoring water and salt movement in soils at low solution contents. Soll Selence, v.141, p.163-171, 1986.

HEERAMAN, D.A; HOPMANS, J.W.; CLAUSNITZER, V. X-ray computed tomography of plant roots. In Press. Plant and Soll. 1997.

HOPMANS, J.W.; DANE, J.H. Calibration of a dualenergy gamma radiation system for multiple point measurements in a soil. Water Resources Research, v.22, n.7, p. 1109-1114, 1986.

HOPMANS, J.W.; VOGEL, T.; KOBLIK, P.D. X-ray tomography of soil water distribution in one-step outflow experiments. Soll Sclence Society of America Journal, v.56, n.2, p.355-362, 1992.
HOPMANS, J.W.; SELKER, J.S.; HENDRICKX, J.H.M Emerging techniques in vadose zone characterization. IN: Vadose Zone Hydrology: Cutting across disciplines (Parlange, M.B.; Hopmans, J.W., ed.). Oxford University Press. In Press. 1997.

HOUNSFIELD, G.N. Computerized transverse axial scanning tomography. Part I: Description of the system. BrttishJournal of Radiolozy, v.46, p.1016-1022, 1973.

KINNEY, J.H., HAUPT, D.L.; MCHOLS, M.C. BREUNIG, T.M.; MARSHALLJR., G.W.; MARSHALL, S.J. Nuclear Instruments and Methods in Physics Research A 347:480-486. Liu, Y.; Bierck, B.R.; Selker, J.S.; Steenhuis, T,.S.; Parlange, J.-Y., 1993. High intensity $X$-ray and tensiometer measurements in rapidly changing preferential flow fields. Soil Science Society of America Journal, v.57, p.1188-1192, 1994.

MCHOLL, M.J.; GLASS, R.J.; WHEATCRAFT, S.W. Gravity driven infiltration instability in initially dry nonhorizontal fractures. Water Resources Research, v.30, n.9, p.2533-2546, 1994.

OOSTROM, M.; DANE, J.H.; GUVEN, O.; HAYWORTH, J.S. Experimental investigation of dense solute plumes in an unconfined aquifer model. Water Resources Research, v.28, n.9, p.2315-2326, 1992.

PETROVIC, A.M.; SIEBERT, J.E.; RIECKE, P.E. Soil bulk density analysis in three dimensions by computed tomographic scanning. Soll Sclence Society of America Journal, v.46, p.445-450, 1982.

PHOGAT, V.K.; AYLMORE, L.A.G. SCHULLER, R.D. Simultaneous measurement of the spatial distribution of soil water content and bulk density. Soil Science Society of America Journal, v.55, p.908-915, 1991.

SPANNE, P.; JONES, K.W.; PRUNTY, L.; ANDERSON, S.H. Potential applications of synchrotron computed microtomography to soil science. p. 43-57. In: Anderson, S.H.; Hopmans, J.W., ed. Tomography of Soil-Water-Root Processes. SSSA Special Publication Number 36. American Society of Agronomy Inc. Madison, Wisconsin, USA. 1994a.

STEUDE, J.S.; SULLIVAN, C.P.; CHANEY, R.C.; MCKEE, M., O'SHEA, L.E. Tracer experiments in saturated sand by computed tomography and inductively coupled plasme. p. 171-184. In: $K$. Hoddinott and R.O. Lamb (eds.) Proc. Symp. on Physio-Chemical Properties of Soil, Rock, and Related Materials. St. Louis, MO. 29 June 1989. American Society Testing and Materials, Philadelphia, PA. 1989. 
TIDWELL, C.; GLASS, R.J. X-ray and visible light transmission for laboratory measurement of twodimensional saturation fields in thin slab systems. Water Resources Research, v.30, n.11, p.28732882, 1994.

TOLLNER, E.W;; RAMSEUR, E.L; MURPHY, C. Techniques and approaches for documenting plant root development with X-ray computed tomography ; p.115-133. In: Anderson, S.H.; Hopmans, J.W., ed. Tomography of Soil-Water-Root Processes. SSSA Special Publication Number 36. American Society of Agronomy Inc. Madison, Wisconsin, USA. 1994.

VINEGAR, H.J.; WELLINGTON, S.L. Tomographic imaging of three-phase flow experiments. Rev ScL. Instrum., v.58, p.96-107, 1987.

\section{EVALUATION OF VARIOUS INFILTRATION MODELS}

Many approaches have been presented to solve the problem of infiltration of water into a soil. Youngs (1995) reviews the historic development of infiltration theory including the classic solutions based on the Richards equation. For a comprehensive review of analytical and empirical solutions see Kutilek \& Nielsen (1994). A typical use of these parameterized solutions is as fitting models for measured data sets. The optimized parameters serve as a convenient, condensed description of the data and are also used for predictive purposes.

Uncertainty is inherent in model predictions and is caused by several factors including imperfectly known model parameters and potential model error, e.g., errors due to violation of assumptions made in the development of the model. It would seem desirable to report, as a rule, an estimate of uncertainty together with any model prediction. While the model error will in general be unknown, the parameter uncertainty can be estimated in form of confidence intervals if the parameters are obtained by fitting measured data. This estimate is derived from the shape of the objective function in the immediate vicinity of the optimized parameter set and will be precise for a linear fitting model with zero model error and measurement errors that are uncorrelated and normally distributed. Using the Monte-Carlo method, it is possible to obtain the actual distributions of optimized parameters that can be used to evaluate the accuracy of estimated confidence limits for nonlinear fitting models.
We compared infiltration equations in terms of their fitting ability and accuracy of estimated parameter confidence intervals. Specifically, we tested six models for three different levels of simulated measurement errors. The objective was to identify the models that provide the best parameter and uncertainty estimates while converging fast and reliably.

Selection of infiltration equations was necessarily somewhat arbitrary given their great number, however, we included those that we believe to be widely used. Empirical models tested were those proposed by Mezencev (1948) and by Horton (1940). Four models derived from physical considerations were tested: Green \& Ampt (1911), Parlange et al. (1982), Philip (1957), and Swartzendruber (1987). Within the framework of assumptions made in the development of these models, their parameters can be physically interpreted. However, when applied to field-obtained infiltration data, the physically based models are used in a manner effectively similar to the empirical models because a number of the assumptions are typically violated (e.g., uniformity of medium properties and of initial water content). Thus for the purposes of this study, each model's parameter vector was considered unknown and subjected to the fitting procedure.

Reference data free of measurement errors are prerequisite to generating data sets with controlled 'measurement' errors. For this purpose, cumulative infiltration $I$ as a function of time $t$ was predicted using a one-dimensional numerical scheme solving the Richards equation. Assumptions inherent in the simulations include a rigid homogeneous medium, isothermal conditions, and a continuous air phase at atmospheric pressure at all times.

The hydraulic properties of a given soil determine the shape of the respective infiltration curve, possible shapes ranging from significantly curved over the time frame of interest to quickly approaching steady state. To cover two points in the soil hydraulic spectrum, reference data were obtained for a sandy loam and for a clay. For each, two I $(t)$ reference data sets over $5 \mathrm{hr}$ and 20 $\mathrm{hr}$ were obtained and represented by 70 and 140 $(I, t)$ points, respectively. Points were selected so as to be equal-spaced on a $t^{0.5}$-axis, thus providing the highest density at the origin.

Fitting data sets over two different periods ( $5 \mathrm{hr}$ and $20 \mathrm{hr}$ ) was done to identify the 